EPJ Web of Conferences 11, 06008 (2011)

DOI:10.1051/epjconf/20111106008

(C) Owned by the authors, published by EDP Sciences, 2011

\title{
New tools and improvements in the Exoplanet Transit Database
}

\author{
S. Poddaný1 ${ }^{1,2}$, L. Brát ${ }^{3,4} \&$ O. Pejcha ${ }^{3,5}$
}

${ }^{1}$ Astronomical Institute, Faculty of Mathematics and Physics, Charles University

Prague, Prague, V Holešovičkách 2, Czech Republic [poddany@observatory.cz]

${ }^{2}$ Štefánik observatory, Prague, Petř́n 205, Czech Republic

${ }^{3}$ Variable Star and Exoplanet Section of Czech Astronomical Society, Czech Republic

${ }^{4}$ Altan observatory, Pec pod Sněžkou 193, Czech Republic

${ }^{5}$ Department of Astronomy, Ohio State University, Columbus, USA

\begin{abstract}
Comprehensive collection of the available light curves, prediction possibilities and the online model fitting procedure, that are available via Exoplanet Transit Database became very popular in the community. In this paper we summarized the changes, that we made in the ETD during last year (including the Kepler candidates into the prediction section, modeling of an unknown planet in the model-fit section and some other small improvements). All this new tools cannot be found in the main ETD paper.
\end{abstract}

\section{Introduction}

The Exoplanet Transit Database (ETD) is still in progress. Each user can find here transit predictions for all transiting planets and their published data with useful references. Almost 2 thousand light curves mainly from the well experienced amateur exoplanet observers are divided into different groups according to their data quality index. The mid-transit times, transit depths and the length of the transits are displayed in online generated O-C plots.

Comprehensive collection of an available light curves, prediction possibilities and the online model fitting procedure, that are available via ETD server ${ }^{1}$ became very popular in the exoplanet community and amateurs all around the World. Many papers that use the services of ETD appeared during this year (see Campo et al 2010; Burke et al 2010; Maciejewski et al 2010a, 2010b, 2010c; Krejčová, Budaj \& Krushevska 2010; Vereš et al 2009).

\footnotetext{
${ }^{1}$ http://var2.astro.cz/ETD/index.php
} 
These are the reasons why we continue to improve the database to be more and more useful for the community. Within two years of operation of the database, we made several changes especially in the prediction and model fit section of the ETD. Most of these changes have been made at the request of our users. This main changes and also many other smaller ones cannot be found in the main ETD paper Poddanýet al 2010, which describes the various components of the database.

\section{New tools in the ETD}

During this summer a new section KEPLER Candidates had been added to the ETD. This section contains 311 Kepler candidates ${ }^{2}$ issued in June 2010 (Borucki et al 2010). Orbital periods of some candidates on this list were derived from preliminary results. Therefore the true orbital period of some candidates could be significantly different. This can cause false transit predictions in the ETD. When we compute the transit duration we assume host stars are near solar main sequence. The stellar mass is then proportional to stellar radius. For all candidates we farther assume inclination to be 86.4 that is an average inclination of transiting planets known in June 2010. Predicted individual transit duration may in some cases differ from the true value from minutes to hours.

Model-fit data section was extended to be able to fit data of an "unknown" planet. Our fitting code was developed to derive online mid transit time, depth and duration already known planets. Hence the initial values and the impact parameter of an "unknown" planet is required. In spite of this restriction this code could be helpful for teams observing some exoplanet candidates, brown dwarfs ...

Prediction section was extended to be more helpful in planing individual observing campaigns. Now every observer can define its own observing time span directly in the prediction section.

Derived system geometry is newly displayed after successfully processing the light curve into the ETD. The planet radius is directly computed from the depth of the transit. Orbit inclination is computed with respect to the star radius uncertainty and the semi major axis, which are usually dominant sources of resulted uncertainty of the inclination.

\section{Future upgrades}

The number of records in the database grows very quickly. It will be necessary to develop a filter in displaying $\mathrm{O}-\mathrm{C}$ plots. Today situation causes (light curves with the best quality as well as the light curves with very poor quality, are pictured into one plot) that possible TTV effects, that should be visible in our O-C plots, are blinded with the huge number of poor quality data. Planning displaying $\mathrm{O}-\mathrm{C}$ plots according to their data quality index should remove this effect.

Most of the data that are submitted to ETD are in Heliocentric Julian Day (HJD) others in Barycentric Julian Day (BJD) (if BJD is used you can now find only a notice in the record form). This discrepancy also causes blending of possible TTV effects. Hence we plan to use BJD for all light curves in the database.

\footnotetext{
${ }^{2}$ Identification of objects are from http://archive.stsci.edu.
} 


\section{Conclusions}

The ETD is still in progress. We try to improve this database to be more and more useful for the community. We are open to suggestions and requirements of individual users.

Acknowledgements. The ETD is maintained by the Czech Astronomical Society. This investigation was supported by the Grant Agency of the Czech Republic, Grant No. 205/08/H005. We also acknowledge the support from the Research Program MSM0021620860 of the Ministry of Education.

\section{References}

Borucki, W., J., et al 2010, submitted to ApJ, arXiv:1006.279

Poddaný, S., Brát, L.,\& Pejcha O. 2010, New Astron., 15, 297

Campo, C. J., et al 2010, ApJ submited

Burke, C. J., et al 2010, ApJ, 719, 1796-1806

Maciejewski G., et al 2010, MNRAS submited

Maciejewski G., et al 2010, Poster contribution to Detection and Dynamics of Transiting Exoplanets (OHP2010)

Maciejewski G., et al 2010, MNRAS, 407, 4, 2625-2631

Krejčová, T., Budaj, J.,\& Krushevska, V. 2010, arXiv:1003.1301v2

Vereš, P. et al 2009, Contributions of the AO Skalnaté Pleso, 39, 1, 34-42 